\title{
Continuously Variable Transmission Using Quadric Crank Chains and Ratchets
}

\author{
T. Yukawa ${ }^{1}$ \\ ${ }^{1}$ Department of Mechanical Engineering \\ Iwate University \\ 4-3-5 Ueda, Morioka, Iwate, 020-8551 (Japan) \\ Phone/Fax number: +81-19-621-6403, e-mail: yukawat@iwate-u.ac.jp
}

\begin{abstract}
This paper describes the continuously variable transmission (CVT). Generally, CVTs are classified as belt-type or toroidal CVTs, and each CVT is basically composed of two parts such as the V-belt and pulley, or friction wheels. In the belt-type CVT, the pulley is driven by a belt placed between two (left and right) circle boards in the pulley, while in the toroidal CVT, two rollers rotate under the condition being pushed by strong compression power. Since these conventional CVTs use friction force, their energy transfer efficiency might be inferior. Furthermore, although these CVTs require precise structures and processing, they make noise, and are not durable. Consequently, we propose a new structural CVT in this paper.
\end{abstract}

\section{Key words}

Continuously Variable Transmission, Quadric Crank Chain, Link Mechanism, Cam, Ratchet

\section{Introduction}

The power transmission mechanism between the input side and the output side in vehicles or turbines includes the gear transmission mechanism, the traction mechanism, friction belts, pulleys, the drive shafts, and the torque converter, etc. The transmission mechanism between the engine side (input side) and the wheel shaft side (output side) is used to change not only the power (force, torque) but also the rotation speed (Fig. 1).

There are two main types of variable transmissions: the multistage type of gear transmission and the continuously variable transmission (CVT) [1]. Since the CVT can continuously change the gear ratio, in theory it should have high transmission efficiency. However, in the conventional CVT, there is significant power loss because the CVT mechanism is a conduction mechanism with friction power occurring under high pressure at the contact points between transmission mechanisms. That is, the internal forces generated by the friction power at the contact points do not cause any work loads, but they cause the dissipation energy.

We here propose a new type of CVT to compensate for the loss that originates in this high pressure [2]-[4]. In this mechanism, which makes use of closed loop-like links of a quadric crank chain, there is a symmetrical arrangement of two crank-rocker mechanisms, which are composed primarily of a closed loop consisting of four links. The type of the movement in each crank-rocker mechanism depends on the relationship between the lengths of the links. In the transmission, the rotation mechanism of the crank serves as the input function, and the rocker mechanism serves as the function of the output side. Then, the power which is generated by the back- and-forth angular movement of the rocker is transferred to the outside rotation mechanism of a output internal gear which connects to the wheel mechanism such as a tire. In the crank-rocker mechanism, the speed ratio can be changed without stage by changing the length of the crank continuously. The ratchet mechanism is used to transmit power from the movement of the rocker to the above-mentioned output rotation mechanism by means of a pawl installed in each rocker, which intermeshes alternately to the ratchet mechanism. (The ratchet may be changed to the one-way clutch in the future). Since this proposed mechanism is not friction-generated under high pressure conditions, it is thought that the transmission efficiency will be greater.

\section{Conventional CVT mechanisms}

The conventional belt-type CVT is a mechanism that transmits power with a V-belt and two pulleys, as shown in Fig. 2. The part where the right and left pulleys are in contact with the belt has the shape of a taper. The position at which the belt hangs relative to the pulleys changes continuously along the direction of the rotation axis, and the distance between the two pulleys shortens or lengthens. That is, the gear ratio can be changed smoothly by controlling the effective diameter of the pulley to contact with the V-belt to transmit the force and torque. Until now, this type of CVT was generally used in small cars whose engine generates only a low torque. Fig. 2(a) shows the case of low gear ratio, and Fig. 2(b) shows the case of a high ratio.

The toroidal CVT shown in Fig. 3 is another type of conventional CVT [5]-[11]. In the toroidal CVT, a power roller is placed between the input and output disks. The points of contact between both of input and output disks and the power rollers are changed as the inclination angle of the power roller is changed along the rotation axis by another exterior force in order to change the speed ratio. This type of CVT is used for a comparatively big type of vehicles with an engine that can generate high power and torque. Fig. 3(a) shows the case in the situation of a speed reduction gear ratio (low ratio), and Fig. 3(b) shows the situation of a multiplying gear ratio (high

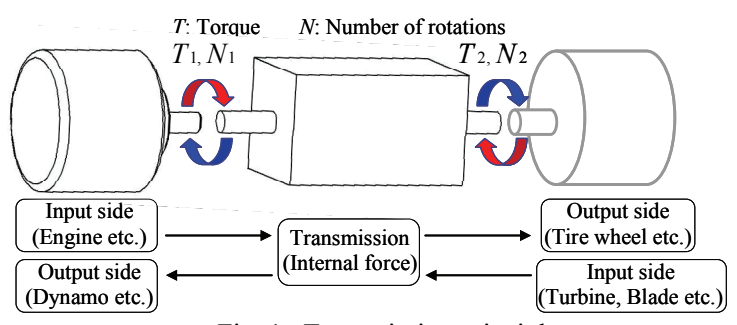

Fig. 1. Transmission principle 
ratio). The toroidal CVT transmits power through the traction oil, which prevents the roller and the disks from wearing out; this helps to maintain performance in lubrication, cooling and transmission by fluid. However, the traction oil solidifies like glass under high contact pressure. Power between the disks and the roller is transmitted through the film of oil that forms.

\section{Quadric Crank Chain}

The structure of a quadric crank chain is shown in Fig. 4. In Fig. 4(a), the lengths of links a, b, c and d are different, and each link is connected by a rotation joint. The link mechanism becomes a crank-rocker mechanism if the condition of the four link lengths is satisfactory. It is assumed that it is possible to rotate the shortest link, a, which is the crank, completely around the rotation axis A. Link $b$, the connecting rod, transmits the movement of link a to the link c. Link c is a rocker whose shuttling corner moves by centering on rotation axis $\mathrm{D}$. When the link $\mathrm{d}$, which is adjoined to rotation joint $\mathrm{A}$, is fixed, the links of the quadric crank chain forms a crank-rocker mechanism. In a precise sense, there is a relationship between the lengths of the different links.

In the quadric crank chain, there are also another three kinds of movements: i) a double-crank mechanism and ii) a parallelogram mechanism, which are continuously in motion, and iii) a double-rocker mechanism whose motion is not continuous. The condition that the movement of the crank-rocker mechanism is appropriate if and only if the sum of the lengths of the shortest link, a, and another one of links is smaller than the sum of the lengths of the other two links [2]-[4], [12]. To achieve the movement as the crank-rocker (lever-crank) mechanism, it is necessary to derive the limitation condition in the length of four links. We consider about the length of each link in the state when the link c comes to the right side of edge, as shown in Fig. 4(b). In $\triangle \mathrm{AC}_{1} \mathrm{D}$, the inequality

$$
a+b<c+d
$$

is satisfied, and in $\Delta \mathrm{AC}_{2} \mathrm{D}$, about the length of each link in the state when the link c comes to the left side of edge,

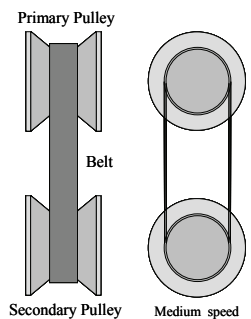

(a)

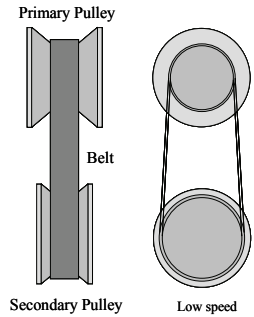

(b)
Fig. 2. Belt CVT

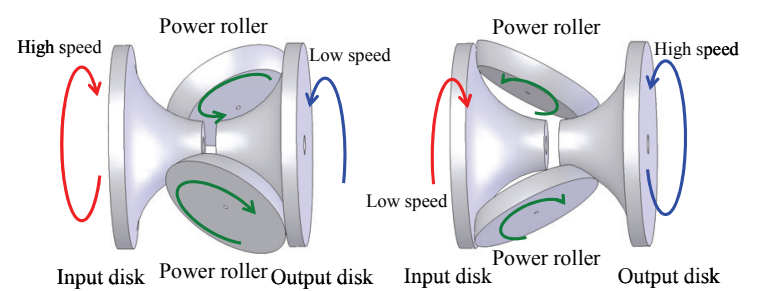

(a)

Fig. 3. Toroidal CVT

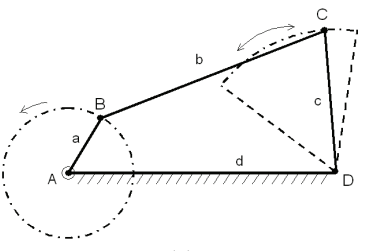

(a)

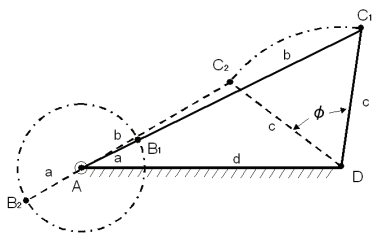

(b)

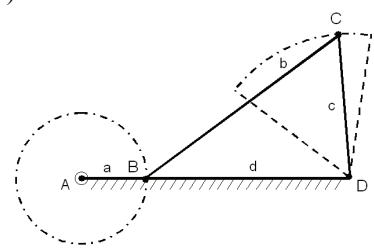

(c)

Fig. 4. Quadric crank chains

as shown in Fig. 4(b), the inequality

$$
b-a+c>d
$$

that is,

$$
a+d<b+c
$$

is satisfied. As shown in Fig. 4(c), in $\triangle \mathrm{BCD}$, the inequality

$$
d-a+b>c
$$

that is,

$$
a+c<b+d
$$

is satisfied, at the same time, the inequality (1) is also satisfied in this case. The inequality (2)' can also be satisfied when the point B comes to the other side for the center point A in Fig. 4(c). Consequently, the shortest link a can rotate if and only if the sum of the lengths of the shortest link a itself and the other one link is smaller than the sum of the lengths of the other two links [2]-[4], [12].

\section{CVT using Quadric Crank Chains}

\section{A. Fundamental Principle}

The outline of a CVT using quadric crank chains is shown in Fig. 5 (a). We named this transmission the LCVT, that is, the CVT consisting of "Links". Two identical quadric crank chains, abcd and a'b'c'd', are

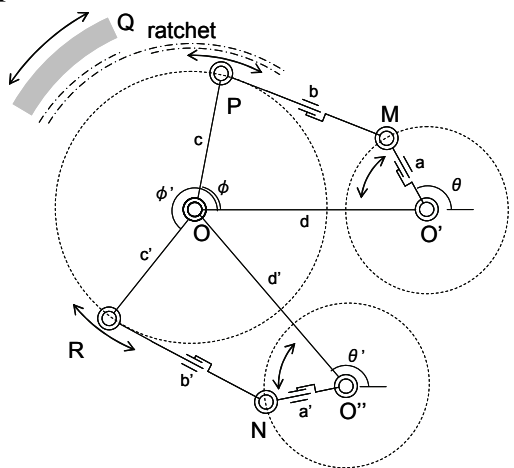

(a) The case in which the axis O' does not correspond to the axis O"

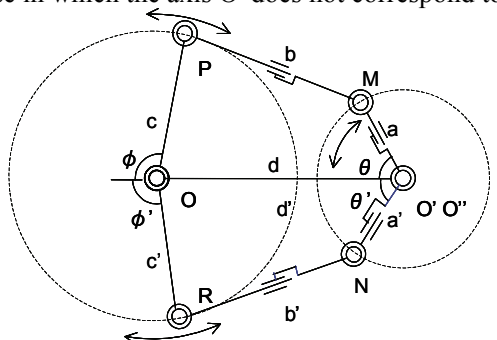

(b) The case in which the axis $\mathrm{O}^{\prime}$ corresponds to the axis $\mathrm{O}^{\prime \prime}$ Fig. 5. Fundamental structures of $\mathrm{L}-\mathrm{CVT}$ 


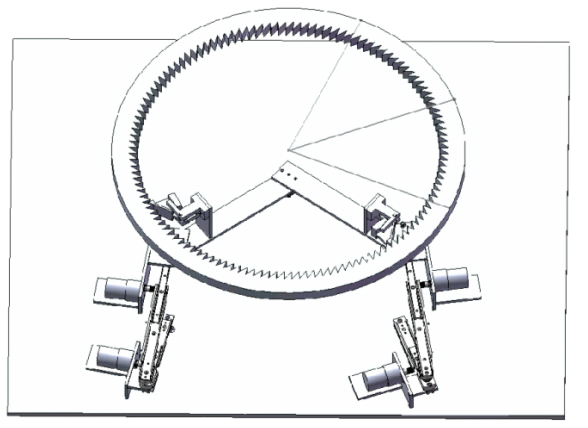

Fig. 6. Fundamental 3D structure of $\mathrm{L}-\mathrm{CVT}$

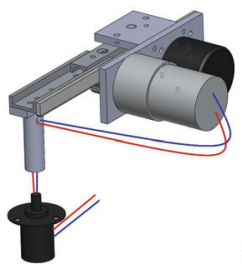

(a) Crank

(Link a) (Link b)
Fig. 7. Crank and connecting rod

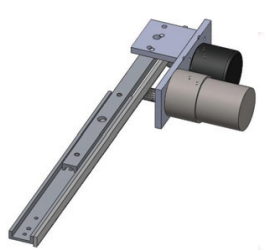

(b) Connecting rod Link b)

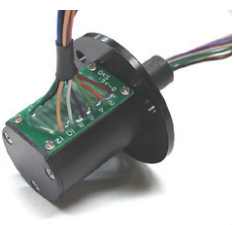

Fig. 8. Collector ring placed symmetrically, and rotation axes $\mathrm{O}^{\prime}$ and $\mathrm{O}^{\prime \prime}$, which are input sides, may synchronize with each other, mechanically. Fig. 5 (b) shows the case in which the axis $\mathrm{O}^{\prime}$ corresponds to the axis $\mathrm{O}^{\prime}$. Joints $\mathrm{P}$ and R swing when links a and a' rotate. With respect to the movement of the rocker, its speed differs on the initial and the return trips, and its acceleration is therefore not constant. Excepting a moment when the initial and return trips of the rocker switch, the acceleration of the rocker should be lost. In other words, the speeds of the initial and return trips should remain constant, and both speeds (the absolute value of the velocity) should be the same. It is necessary to set the expansion and contraction movement of sticks $\mathrm{b}$ and $\mathrm{b}$, which are jointed between crank a and rocker $\mathrm{c}$ to provide this movement and achieve an equiangular angle movement with constant velocity. As a result of the expansion and contraction, angle $\phi\left(=\phi^{\prime}\right)$ of link $\mathrm{c}\left(=\mathrm{c}^{\prime}\right)$ becomes a triangular wave, and the derivative value angle speed $\mathrm{d}_{\phi} / \mathrm{dt}\left(=\mathrm{d} \phi^{\prime} / \mathrm{dt}\right)$ of link $\mathrm{c}\left(=\mathrm{c}^{\prime}\right)$ becomes a rectangular wave for time axis. As a result, $\mathrm{P}$ and $\mathrm{R}$ repeat their motion with equal velocity in the direction of order, and the opposite direction every half cycle. Power is transmitted only to one direction in the swing movement of the link using a quadric crank chain, so it is possible to design the mechanism to conduct power
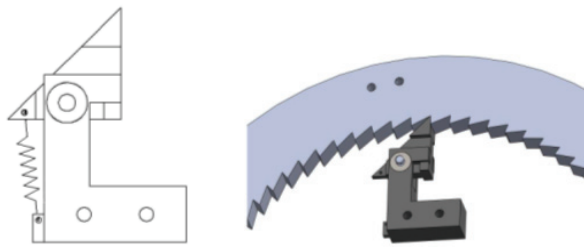

(a) Mechanism of the ratchet (b) Ratchet and internally-toothed gear Fig. 9. Ratchet, and internally-toothed gear

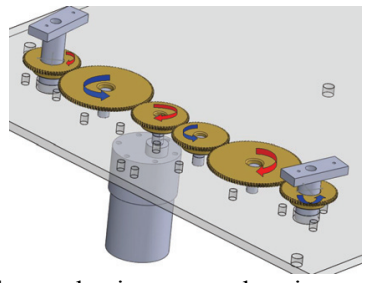

Fig. 10. The mechanism to synchronize two input cranks

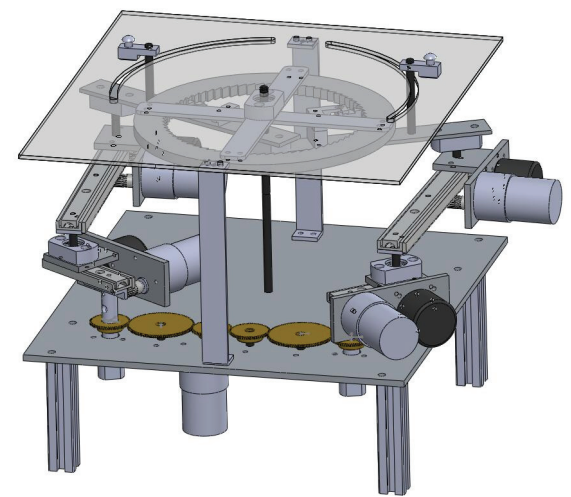

(a) 3D structure of the L-CVT

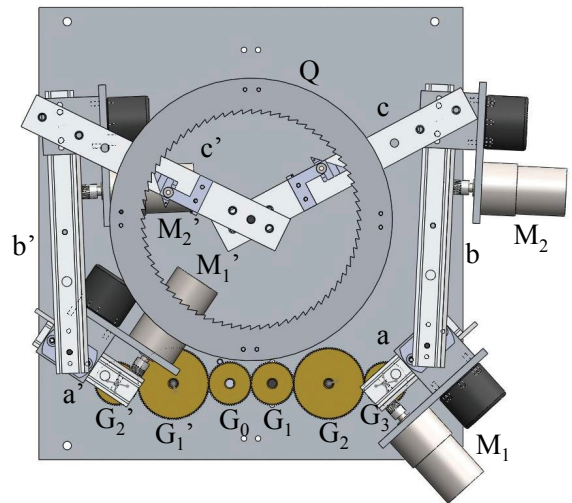

(b) Top view

Fig. 11. Entire mechanism of $\mathrm{L}-\mathrm{CVT}$

continuously using at least two quadric crank chains. As one means of achieving continuous conduction of power, if the rotation phase of synchronizing links $\mathrm{c}$ and $\mathrm{c}^{\prime}$ is reversed by 180 degrees, then the swing phase of $\mathrm{P}$ is shifted to $\mathrm{R}$ by just a half-phase corresponding to a halfcycle. As another means, the rotations of links a and a' can be reversed though an idle gear or similar mechanism.

Next, we examine the ratchet mechanism, which is used to make the power generated by the swing of rotation joints $\mathrm{P}$ and $\mathrm{R}$ be conducted in only one direction. (Another two ratchets and output internal gear operating in the opposite directions will be used to switch the normal and reverse directions of the rotation if needed). When the ratchet moves along the opposite direction for the output gear, no power is transmitted. Therefore, the output gear (parts Q in Fig. 11) through the ratchet can be rotated at a constant speed. Under this method, whole system becomes a deceleration mechanism that can adjust the relationship between the angle speed $\mathrm{d} \theta / \mathrm{dt}(=$ $\left.\mathrm{d} \theta^{\prime} / \mathrm{dt}\right)$ of links a and $\mathrm{a}^{\prime}$, and the angle speed $\mathrm{d} \phi / \mathrm{dt}(=$ $\mathrm{d} \phi^{\prime} / \mathrm{dt}$ ) of links $\mathrm{c}$ and $\mathrm{c}^{\prime}$, by the expansion and contraction of links $b$ and $b$ ' with the prismatic joint mechanism.

\section{B. Fundamental Structure of L-CVT}

A three-dimensional chart of the basic structure of the L-CVT is shown in Fig. 6. Links d and d' (Fig. 5) in the quadric crank chains are fixed on a base. The rack gear and motor, which expand and contract due to the driving power of the motor, are mounted on the slide rail at crank a (Fig. 7(a)) and link b (Fig. 7(b)), which connects the crank and the rocker. In addition, a pawl for the ratchet is installed in link c. The pawl is intermeshed with the 
large-diameter output gear (as shown in Fig. 6) which is also an internal gear.

A collector ring (rotation electrode) (Fig. 8) is used to supply electric power to the motor $\mathrm{M}_{1}\left(\mathrm{M}_{1}{ }^{\prime}\right)$ (Fig. 11(b)). Since the collector rings are arranged in the same rotation axes $\mathrm{O}^{\prime}$ and $\mathrm{O}^{\prime \prime}$, the power supply to the motors $\mathrm{M}_{1}$ and $\mathrm{M}_{1}{ }^{\prime}$ can be supplied by the lead line, which passes through the cave part of the cylinder axis of the slipping ring and the midair portion of the axes. As a result, electric power to the motor is supplied from the outside without twisting the lead line.

\section{Ratchet Mechanism and the Internal Gear}

The ratchet mechanism for transmission from the swing of rotation joints to the internal gear as an output is shown in Fig. 9(a). A three-dimensional diagram of the ratchet mechanism and an enlargement of the ratchet intermeshed with the output internal gear is shown in Fig. 9(b). Each pawl of the ratchet installed in the rockers c and $c^{\prime}$ intermeshes with the large-diameter internal gear (see Fig. 11(b)). The pawl of the ratchet has a structure which transmits force in only one direction. When the ratchet is idled with the internal gear, the teeth of the ratchet go over without any powers, and the pawl then returns to the equilibrium position according to the force of restitution of the spring mounted on the ratchet. For the large-diameter internal gear as an output gear, the diameter of the outer circumference is $200 \mathrm{~mm}$, the addendum circle is $150 \mathrm{~mm}$, thickness is $8 \mathrm{~mm}$, and the number of teeth is 72 .

\section{Transmission Mechanism in the Axis of the Generating Machinery Input Side}

Fig. 10 shows the transmission mechanism in the axis of the generating machinery input side, which synchronizes rotation axis $\mathrm{O}^{\prime}$ with rotation axis $\mathrm{O}^{\prime \prime}$ (as shown in Fig. 5(a)). In the prototype model, a motor is used as an input so that the two cranks can be mechanically synchronized, and this motor synchronizes the rotation of the two cranks precisely through timing belts or spur gears $\left(\mathrm{G}_{0}, \mathrm{G}_{1}, \mathrm{G}_{2}, \mathrm{G}_{3}, \mathrm{G}_{1}{ }^{\prime}\right.$, and $\mathrm{G}_{2}{ }^{\prime}$, as shown in Fig. 11(b)). In order for the power to be transmitted to the two cranks, one of the cranks must be reversed through an idle gear.

\section{E. L-CVT System}

Fig. 11(a) shows the entire mechanism of the L-CVT system in which the ratchet mechanism, the internal output gear, and the generating machinery input side have been added. The output internal gear is fixed to the top and bottom with holders installed on the main body so that it will not move, except for its rotation. The total size of the system is $300 \mathrm{~mm}$ in width, $320 \mathrm{~mm}$ in length, and $165 \mathrm{~mm}$ in height. From the top view of L-CVT as shown in Fig. 11(b), it is understood that this mechanism corresponds to Fig. 5(a).

\section{F. $\quad$ Another Mechanism}

There are other methods to expand and contract links such as cranks a, a', and connecting rods $b, b^{\prime}$ for the

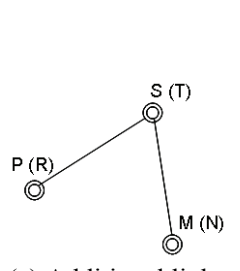

(a) Additional link

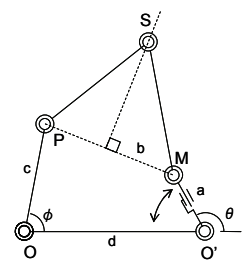

(b) Five-linkage

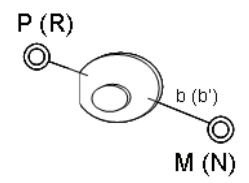

(c) $3 \mathrm{D}$ cam

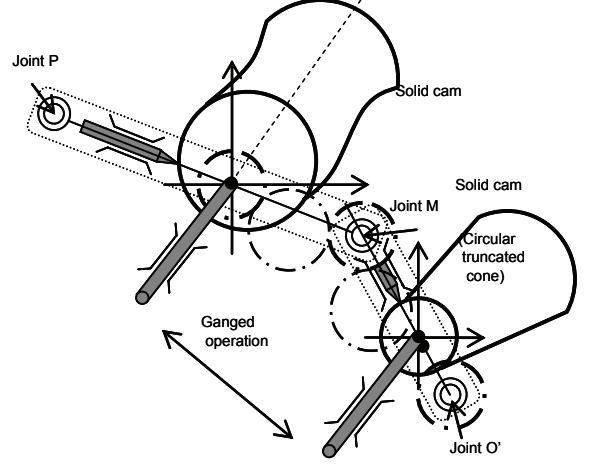

(d) Mounting of 3D cam

Fig. 12. Other mechanisms for extensible link

expand and contract mechanism, and are shown in Fig. 12 (a), (b)). Under one such method, a new joint $(\mathrm{S}(\mathrm{T})$ in Fig. 12(a)) is rotated by including a new link between $\mathrm{O}^{\prime} \mathrm{M}\left(\mathrm{O}^{\prime \prime} \mathrm{N}\right)$ or PM $(\mathrm{RN})$. In this mechanism which makes use of closed five links [13], there is a redundant degree of freedom, and it is shown in Fig. 12(b). Another possible method is to put on a solid cam after cutting the link b (Fig. 12(c)). As a result, the swing of $\mathrm{P}(\mathrm{R})$ becomes a uniformly angled velocity motion using these mechanism at half-cycle intervals. When solid cams are used, a uniformly angled velocity motion of link c can be achieved in a certain gear ratio based on the length of links $\mathrm{a}$ and $\mathrm{c}$ constrained by a curved surface corresponding to the circumference of the solid cams along the direction of the rotation axis (Fig. 12(d)). Then, joints $\mathrm{P}$ and $\mathrm{R}$ move with uniform motion by the expansion and contraction of links $\mathrm{a}, \mathrm{a}^{\prime}, \mathrm{b}$, and $\mathrm{b}^{\prime}$ with the cam. This method has the advantage that it is easy to design a variety of curved circumferences in the vertical cross-sectional surface respect to the rotation axis. The cam can respond to an arbitrary deceleration ratio by sliding along the direction of the rotation axis.

With respect to the power source for changing the gear ratio, an additional driving energy is needed to move the cam along its rotation axis and change the speed of the output gear. Still, this method may have the advantage because the power and torque need not be basically transmitted by friction force.

\section{Simulations}

\section{A. Simulation with Numerical Analysis Software}

Using the numerical analysis software, MATLAB, we simulated a situation in which the crank-rocker mechanism moves back and forth in an angular movement at a uniform velocity. The mechanism corresponding to this simulation is a four-link, quadric crank chain, as shown in Fig. 4. The conditions of the link lengths in the simulation were $a=15, c=30$ and $d=$ 50 (dimensionless). The length of the link of connected sticks, $b$, is the distance between the endpoint $\mathrm{B}(\mathrm{M})$ of 


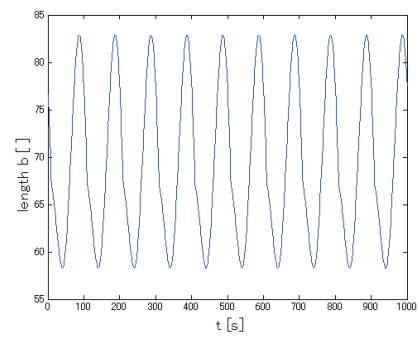

(a) The length of link $\mathrm{b}(a=15)$

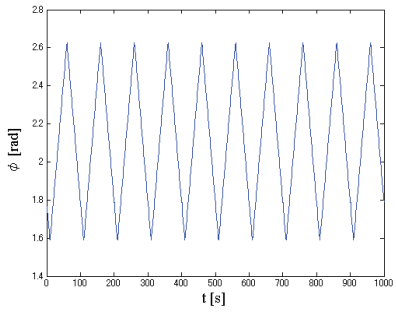

(c) The angle of link c $(a=15)$

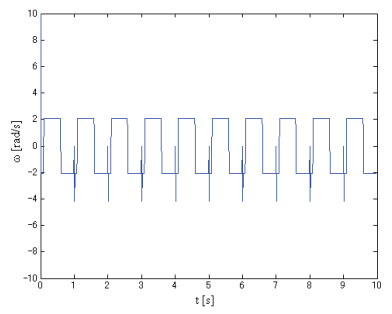

(e) The angular velocity of link c $(a=15)$

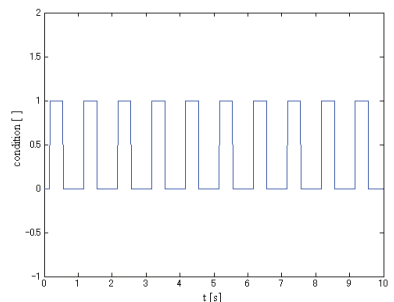

(g) The formation condition $(a=15)$ (h) The formation condition $(a=20)$ Fig. 13 The simulation results

link a and the endpoint $\mathrm{C}(\mathrm{P})$ of link $\mathrm{c}$. The displacement of link c for time is assumed to be $c(t)$, and it is assumed that the link b rotates by $\theta(t)=2 \pi \cdot t$. Let the time when $\phi$ becomes a maximum value, and a minimum value be $t_{l}$ and $t_{2}$, respectively. The following expression:

$$
\begin{aligned}
& \phi_{\max }=\pi-\cos ^{-1}\left[\frac{c^{2}+d^{2}-\left\{a-b\left(t_{1}\right)\right\}^{2}}{2 c d}\right] \\
& \phi_{\min }=\pi-\cos ^{-1}\left[\frac{c^{2}+d^{2}-\left\{a+b\left(t_{2}\right)\right\}^{2}}{2 c d}\right] \\
& \theta_{1}\left(t_{1}\right)=\cos ^{-1}\left[\frac{d^{2}+\left\{a-b\left(t_{1}\right)\right\}-c^{2}}{2 d\left\{a-b\left(t_{1}\right)\right\}}\right] \\
& \theta_{2}\left(t_{2}\right)=\cos ^{-1}\left[\frac{d^{2}+\left\{a+b\left(t_{2}\right)\right\}-c^{2}}{2 d\left\{a+b\left(t_{2}\right)\right\}}\right]
\end{aligned}
$$

can be given. Set

$$
k_{1}=\frac{\phi_{\max }-\phi_{\min }}{\pi}
$$

and

$$
k_{2}=\frac{\phi_{\max }-\phi_{\min }}{\pi},
$$

then, the following relationships (10)-(12) are derived. When $2 \pi n \leqq \theta(t)<\theta_{2}+2 \pi n$ is satisfied,

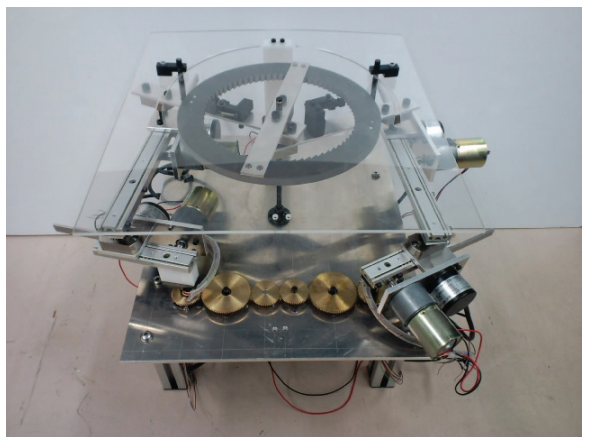

Fig. 14. The photograph of L-CVT

then,

$$
\phi(t)=\phi_{\max }+k_{2}\left\{\theta(t)+\pi-2 \pi n-\theta_{2}\right\}
$$

is given, when $\theta_{2}+2 n \pi \leqq \theta(t)<\theta_{2}+\pi+2 n \pi$ is satisfied,

$$
\phi(t)=\phi_{\min }+k_{1}\left\{\theta(t)-2 \pi n-\theta_{2}\right\}
$$

is given, and, when $\theta_{2}+\pi+2 n \pi \leqq \theta(t)<2 \pi n+2 \pi$ is satisfied,

$$
\phi(t)=\phi_{\max }+k_{2}\left\{\theta(t)-2 \pi n-\theta_{2}-\pi\right\}
$$

is given. $(n=0,1,2, \ldots)$.

The expansion and contraction of the connecting rod $b$ was analyzed, and are shown in Fig. 13. Fig. 13 shows time changes in the swing angle of rotation joint $\mathrm{C}(\mathrm{P})$. The horizontal axis shows time. In Figs. 13(a), (b), the vertical axis shows the changes in the length of link b. It is understood that the length of connecting rod b has a width of expansion and contraction of (a) 58-83 ((b) 5686). Fig. 13(c), (d) show the time responses of the joint angle of link c. Fig. 13(e), (f) show the time responses of the angular velocity of link c. From these figures, it can be determined that connecting rod $b$ can make a uniform angular velocity movement of the link c. Fig. 13(g), (h)
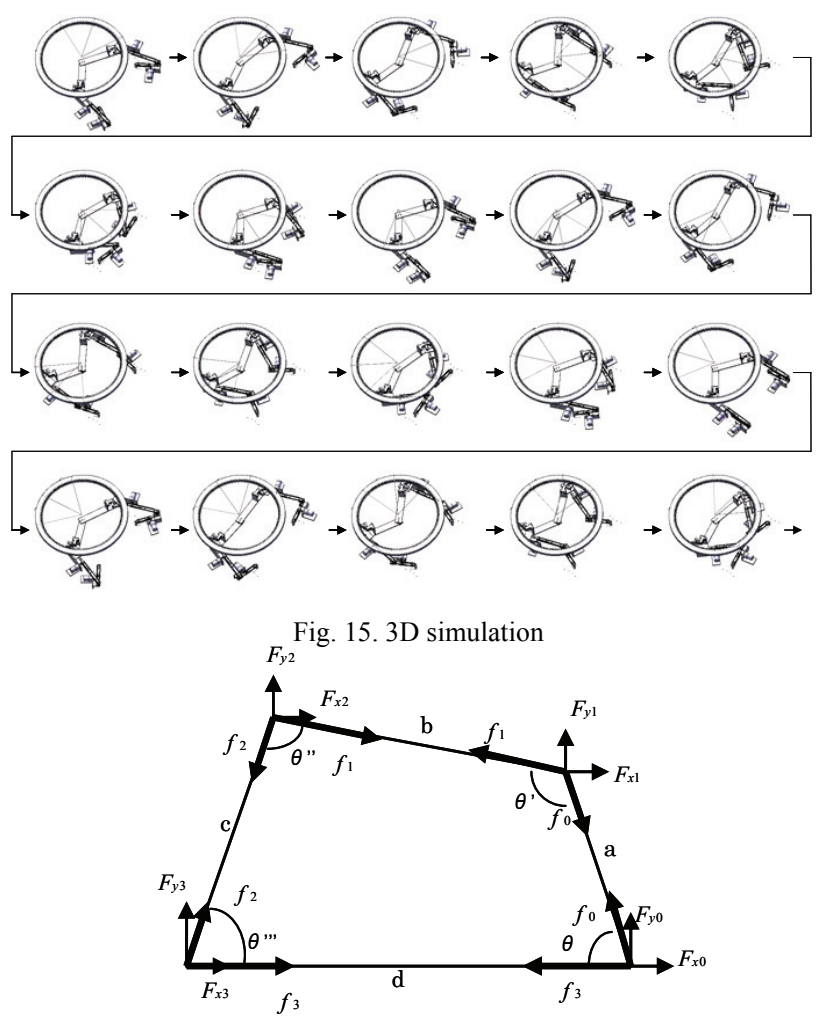

Fig. 16 The force relationship in the quadric crank chain 


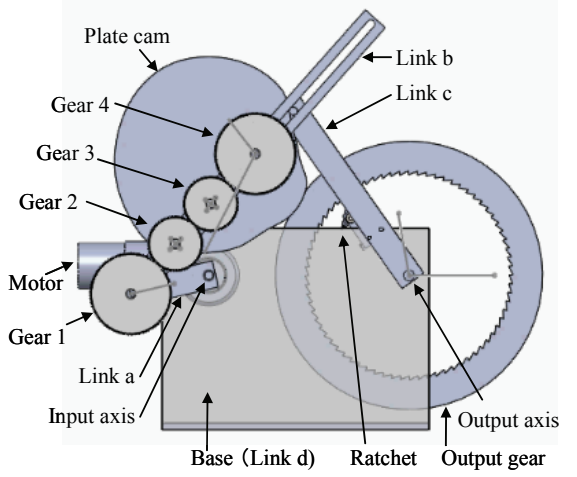

Fig. 17. L - CVT with a solid cam

show the formation condition to satisfy the inequalities (1), (2)', and (3)'. These figures show that the conditions to form the lever crank mechanism is approved when the value in these figures equals one, and is not approved in zero.

\section{B. Simulation using $3 D$ CAD Function}

The operation of the L-CVT mechanism shown in Fig. 6 (Fig. 11(a)) was confirmed using the simulation function of three-dimensional CAD software (SolidWorks). The rotation motor was arranged in center $\mathrm{O}^{\prime}, \mathrm{O}^{\prime \prime}$ rotation of two cranks. The photograph of the LCVT is shown in Fig. 14. The result of the simulation is shown in Fig.15. The rotation trajectory of the output internal gear can be easily understood by additional sectorial lines.

\section{Calculation of the force generated in each link in a quadric crank chain}

Here, the force generated in each link in a quadric crank chain (Fig. 4(a)) is calculated. Define the forces as $f_{0}, f_{1}, f_{2}$, and $f_{3}$, as shown in Fig. 16. Then, the horizontal and vertical elements of the forces applied to each joint are give by

$$
\begin{gathered}
F_{y 0}=f_{0} \sin \theta \\
F_{x 0}=-f_{0} \cos \theta \\
F_{y 1}=-f_{0} \sin \theta+f_{1} \sin \left(\theta^{\prime}-180^{\circ}+\theta\right) \\
F_{x 1}=f_{0} \cos \theta-f_{1} \cos \left(\theta^{\prime}-180^{\circ}+\theta\right) \\
F_{y 2}=-f_{2} \sin \theta^{\prime \prime \prime}-f_{1} \cos \left(\theta^{\prime \prime}-90^{\circ}+\theta^{\prime \prime \prime}\right) \\
F_{x 2}=-f_{2} \cos \theta^{\prime \prime \prime}+f_{1} \sin \left(\theta^{\prime \prime}-90^{\circ}+\theta^{\prime \prime \prime}\right) \\
F_{y 3}=f_{2} \sin \theta^{\prime \prime \prime} \\
F_{x 3}=f_{2} \cos \theta^{\prime \prime \prime} .
\end{gathered}
$$

Eliminating the forces $f_{0}, f_{1}, f_{2}$, and $f_{3}$ from (13)-(20), the following relations

$$
\begin{aligned}
& F_{x 2}+F_{x 3}=\left(F_{x 0}+F_{x 1}\right) \frac{\cos \left(\theta^{\prime \prime}+\theta^{\prime \prime}\right)}{\cos \left(\theta+\theta^{\prime}\right)} \\
& F_{y 2}+F_{y 3}=\left(F_{y 0}+F_{y 1}\right) \frac{\sin \left(\theta^{\prime \prime}+\theta^{\prime \prime \prime}\right)}{\sin \left(\theta+\theta^{\prime}\right)}
\end{aligned}
$$

are given.

\section{Conclusions}

We have proposed an L-CVT in which the link of the quadric crank chain, the cam mechanism and the ratchet mechanism were mounted. Since the proposed method is not based on friction conduction, it provides a mechanism that creates no noise and no slip [14], is durable, and offers high transmission efficiency. Furthermore, the appropriate geometrical condition of the links of the quadric crank chain was identified by simulation.

In future work, we will install the solid cam to the prototype model, and evaluate the transmission efficiency of a CVT. It is a valid point that the synchronization between the rotation of the cam and the one of the crank at the input axis can be taken mechanically without any electrical control.

\section{Acknowledgement}

This work was supported by Suzuki Foundation (Fiscal-2008 Financial Assistance), and was supported by Fundamental Research Developing Association for Shipbuilding and Offshore (REDAS, Fiscal-2010 Financial Assistance).

\section{References}

[1] Y. Morimoto, "Continuously Variable Transmission, (CVT sagoge), "Grand Prix Publication, (2005), (in Japanese).

[2] T. Yukawa, T. Kumada, G. Obinata, Continuously Variable Transmission Using Quadric Crank Chains, 8th IEEE Int. conf. on Industrial Informatics (INDIN 2010), (2010), pp. 1043-1048.

[3] T. Yukawa, "Fundamental Study of a Continuously Variable Transmission Using Quadric Crank Chains, Cams, and Ratchets, " The 51th Japan Joint Automatic Control Conference, 703, (2008), (in Japanese).

[4] T. Yukawa, "Fundamental Study of a Continuously Variable Transmission Using Quadric Crank Chains, ", 2008 JSME Conference on Robotics and Mechatronics, 2P1-A21, (2008), (in Japanese).

[5] H. Tanaka, “Toroidal CVT, "'Corona publishing, (2000), (in Japanese).

[6] J. Ingvast, J. Wikander, and Christian Ridderstrom, "The PVT, an Elastic Conservative Transmission, "The International Journal of Robotics Research, Vol. 25, No.10, (2006), pp. 1013-1032.

[7] S. Miyata, et al., "Study of the Control Mechanism of a Half-Toroidal CVT during Load Transmission, "J. of Advanced Mechanical Design, Systems, and Manufacturing, Vol. 1, No.3, (2007), pp. 346-357.

[8] J. Kim, H. Yeom, F.C. Park, Y.I.Park, Munsang Kim, “'On the Energy Efficiency of CVT-Based Mobile Robots,", Proc. of the 2000 IEEE RA, (2000), pp. 1539-1544.

[9] P. Setlur, J. Wagner, D. Dawson, and B. Samuels, "Nonlinear Control of a Continuously Variable Transmission (CVT) for Hybrid Vehicle Powertrains, , IEEE Transactions on Control System Technology, vol. 11, no. $1,(2003)$, pp. 101-108.

[10] M. Pesgens, B. Vroemen, F. Veldpaus, M. Steinbuch, "Control of a continuously variable transmission in an experimental vehicle, "ADVANCES IN AUTOMOTIVE CONTROL 2004, Proc. volume from the IFAC Symposium, Salerno, Italy, 19-23, (2004), pp. 71-77.

[11] Y. Itoh, T. Sakaguchi, Development of the Mono Ring CVT, NTN Technical Review No. 73, (2005), pp. 60-65.

[12] S. Inada and H. Morita, "Mechanics, "Ohmsha, First Edition, (2001), (in Japanese).

[13] K. Ogawa, H. Shimojima, Synthesis of Planar MultipleLink Path Generator, The Japan Society of Mechanical Engineers (JSME) 14(68), (1971-02), pp. 164-170.

[14] I. S. Privalov and D. V. Chernyavskii, Method of Exactly Determining Slip in a Friction Transmission, J. of Measurement Techniques Vol. 14, No. 11, (1970), pp. 1688-1689. 